diagnostics in those with metastatic cancer who are elderly, com-morbid or of poor performance status and multidisciplinary discussion including palliative care input may help such decision making.

\section{ATU-6 RESULTS OF RAPID HEPATITIS C TREATMENT SCALE-UP FOR PEOPLE WHO INJECT DRUGSIN TAYSIDE, SCOTLAND}

${ }^{1}$ Christopher Byrne* ${ }^{*}{ }^{1,2}$ John F Dillon. ${ }^{1}$ Molecular and Clinical Medicine, University of Dundee School of Medicine., Dundee, UK; ${ }^{2}$ Dept of Gastroenterology, Ninewells Hospital and Medical School, Dundee, UK

\subsection{6/gutjinl-2021-BSG.47}

In 2017, NHS Tayside rapidly scaled-up treatment among People Who Inject Drugs (PWID) for Hepatitis C (HCV) through novel care pathways. This was to drive the region towards HCV elimination by initiating treatment for 590 PWID and obtaining 540 Sustained Virologic Responses (SVR), over three years. In 2020, Tayside declared elimination of HCV. This study analyses: treatment outcomes for PWID treated from 2017-20 and the proportionate efficiency of treatment pathways.

Demographic and clinical parameters were collected for PWID treated for HCV using Direct Acting Antivirals (DAA) from January 2017-April 2020. Follow-up was censored December 2020. Anonymised data was stored. Descriptive statistics were performed using IBM SPSS 25. SVR was calculated as a proportion of treatments.

HCV treatment was initiated 713 times among 662 PWID. Patients were mostly male (74.2\%), and typically resided in deprived areas $(80.4 \%$ Scottish Index Multiple Deprivation 1 or 2). HCV genotype 3 was most common (56.5\%) followed by 1 (39.3\%). Liver cirrhosis was observed in a minority (9.1\%), and most reported receipt of Opiate Substitution Therapy (77.3\%). 573 (80.4\%) SVRs were observed. Excluding those with no SVR test $(n=89)$, this rises to $91.8 \%$. Highest SVR was observed in the community pharmacy pathway $(89.6 \%)$ but, excluding those without a test, the nurse-led community clinics yielded highest SVR at 96.2\%, followed by pharmacies at $95.6 \%$ (Table 1). Community pharmacies and the needle exchange pathway contributed almost half of all treatment initiations (49\%).

NHS Tayside exceeded its treatment target for the scale-up programme. The data demonstrate high levels of cure across novel pathways implemented in community settings. The data suggests moving HCV treatment from secondary to community care can significantly reduce the burden of $\mathrm{HCV}$ infection among PWID at a regional level.

\section{Posters - highly commended}

\section{Endoscopy}

\section{HTU-1 COMPUTER AIDED CHARACTERISATION OF COLORECTAL POLYPS USING ARTIFICIAL INTELLIGENCE}

\footnotetext{
1,2Rawen Kader*, ${ }^{3}$ Anton Mejias, ${ }^{3}$ Sanjith Hebbar, ${ }^{3}$ Patrick Brandao, 'Shahraz Islam, 1,2 Omer F Ahmad, ${ }^{2}$ Mohamed Hussein, ${ }^{4}$ Vinay Sehgal, ${ }^{4}$ Roser Vega, ${ }^{4}$ Edward Seward, ${ }^{3}$ Peter Mountney, ${ }^{1,2}$ Danail Stoyanov, ${ }^{1,2}$ LaurenceB Lovat. ${ }^{1}$ Wellcome/EPSRC Centre for Interventional and Surgical Sciences (WEISS), UCL, London, UK; ${ }^{2}$ Division of Surgery and Interventional Sciences, UCL, London, UK; ${ }^{3}$ Odin Vision, London, UK; ${ }^{4}$ University College London Hospital (UCLH), London, UK
}

\subsection{6/gutjil-2021-BSG.48}

Introduction Optical diagnosis is the in-vivo prediction of colorectal polyp histopathology. Inter-observer variability amongst endoscopists has limited its application in clinical practice. Artificial intelligence may lead to a new generation of clinical support tools capable of characterising polyps. Research in this field has often relied upon retrospective datasets, which are subject to sample selection bias, and consist of a limited number of images of each polyp.

Our aim was to develop a convolutional neural network (CNN) to characterise colorectal polyps as adenomatous or non-adenomatous using data collected prospectively.

Methods Video data was collected prospectively from colonoscopy procedures at a single centre using Olympus 260 and 290 series scopes. Histopathological classification, location and morphology was recorded for each polyp.

Video sequences of polyps in Narrow Band Imaging (NBI) and NBI-Near Focus (NBI-NF) were extracted. Both imaging modalities were used to increase the generalisability of the $\mathrm{CNN}$. Frames with poor visualisation of the polyp surface texture due to mucus, stool, halation or motion artifact were excluded. The ground truth for each frame was the polyp annotated with a bounding box and labelled with the histopathology.

A ResNet-101 CNN pre-trained on ImageNet was developed to classify the visual appearance of colorectal polyps as adenomatous or non-adenomatous.

Results The final dataset consisted of 371 histologically confirmed polyps (235 adenomas, 77 sessile serrated lesions, 58 hyperplastic, 1 traditional serrated adenoma) from 199 patients with a total of 31,110 video frames annotated. Data was split, as shown in Figure 1, into a training ( $\sim 50 \%)$, validation $(\sim 10 \%)$, and testing dataset $(\sim 40 \%)$ with no overlap of polyps or patients.

On a per-frame analysis, the accuracy of the CNN optical characterisation was 91\%, with a sensitivity of $91 \%$ to

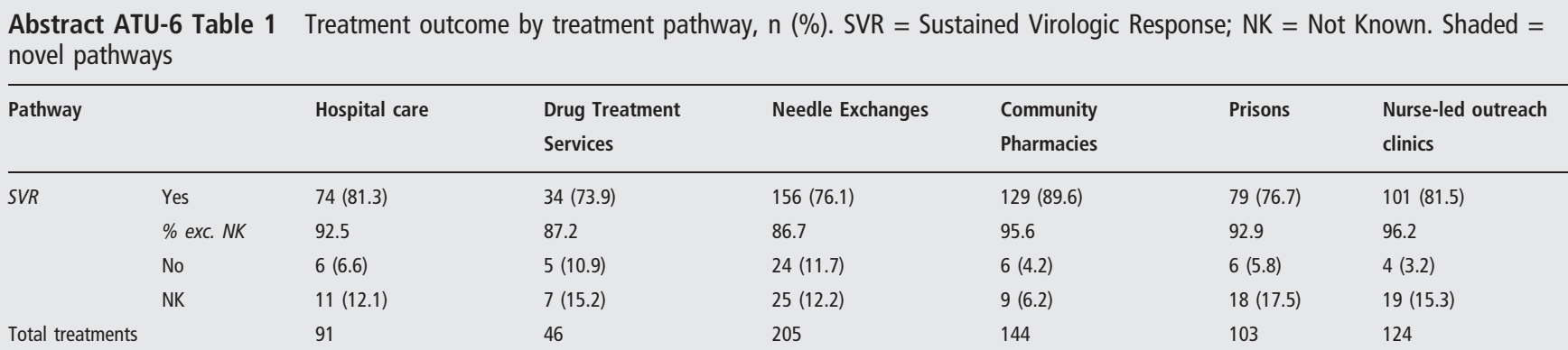




\begin{tabular}{|c|c|c|c|c|}
\hline & Training Dataset & $\begin{array}{c}\text { Validation } \\
\text { Dataset }\end{array}$ & Testing Dataset & $\begin{array}{c}\text { Total } \\
\text { (frames) }\end{array}$ \\
\hline Number of patients & 107 & 14 & 78 & 199 \\
\hline Adenomatous polyp & 122 & 19 & 94 & $\begin{array}{c}235 \\
(21,831)\end{array}$ \\
\hline Hyperplastic polyps & 21 & 2 & 35 & $\begin{array}{c}58 \\
(3,262)\end{array}$ \\
\hline Sessile serrated lesions & 43 & 11 & 23 & $\begin{array}{c}77 \\
(6,017)\end{array}$ \\
\hline $\begin{array}{c}\text { Traditional serrated } \\
\text { adenomatous polyps }\end{array}$ & 1 & 0 & 0 & $1(42)$ \\
\hline $\begin{array}{c}\text { Total number of polyps } \\
\text { (frames) }\end{array}$ & $\begin{array}{c}187 \\
(14,936)\end{array}$ & $\begin{array}{c}32 \\
(1,896)\end{array}$ & $\begin{array}{c}152 \\
(14,320)\end{array}$ & $\begin{array}{c}371 \\
(31,086)\end{array}$ \\
\hline
\end{tabular}

diagnose adenomas and a specificity of $90 \%$. The CNN achieved an area under the curve (AUC) of $97 \%$. On a per polyp analysis, the accuracy of the CNN characterisation was $92 \%$, with a sensitivity of $92 \%$ and a specificity of $93 \%$.

Conclusion The largest annotated dataset of NBI polyp images has been collated for the training and evaluation of artificial intelligence to support optical diagnosis. This work demonstrated the capability of AI to differentiate adenomatous from non-adenomatous polyps in-vitro, with a high level of accuracy.

\section{HTU-2 EUS GUIDED GASTROJEJUNOSTOMY FOR THE MANAGEMENT OF GASTRIC OUTLET OBSTRUCTION: A SINGLE CENTRE EXPERIENCE}

Wei On*, Matthew T Huggett, Bharat Paranandi. Leeds Teaching Hospitals NHS Trust, Leeds, UK

\subsection{6/gutjil-2021-BSG.49}

Introduction There are various modalities of management of gastric outlet obstruction (GOO) namely endoscopic enteral stenting, surgical bypass or placement of a venting gastrostomy tube. Endoscopic ultrasound guided gastrojejunostomy (EUSGJ) via placement of a lumen apposing metal stent has recently emerged as a viable and effective method of managing GOO. We aimed to describe the procedural characteristics, technical and clinical outcomes in patients who underwent EUS-GJ at our institution.

Methods A prospectively collected database of consecutive EUS-GJ procedures from August 2018 to October 2020 at our institution was reviewed retrospectively. All procedures were performed by two expert pancreatobiliary endoscopists. Recorded variables included patient demographics, technical success, clinical success, adverse events, 30-day all cause mortality and follow-up duration.

Results Sixteen patients $(9$ males) with a mean age of 64.5 years old (range 48 - 80 years old) were identified. Malignancy was the predominant aetiology of GOO in our patient cohort $(81.3 \%, \mathrm{n}=13)$. The technical success rate (defined as fluoroscopic and endoscopic confirmation of adequate stent deployment and positioning) was $93.8 \%(\mathrm{n}=15)$, in whom the clinical success rate (defined as toleration of at least liquid diet without vomiting before discharge) was $100 \%$. In the solitary case of technical failure, dislodgement occurred during balloon dilatation of the stent, the defect was endoscopically closed with clips and a duodenal stent was placed. The patient did not experience any adverse consequences post-procedure. Nine patients who required intravenous parenteral nutrition pre-procedure were successfully weaned off following EUS-GJ.
The median follow-up was 109 days (range 5 - 383 days). No adverse events were encountered. One patient required reintervention at day 70. 30-day all cause mortality was 6.3\% $(\mathrm{n}=1)$ and the death was due to malignant disease progression.

Conclusions EUS-GJ is an effective and safe procedure for the management of GOO. It should be considered in appropriately selected patients if there is available expertise.

\section{HTU-3 OPTICAL DIAGNOSIS OF SMALL POLYPS AT COLONOSCOPY VERSUS HISTOPATHOLOGY: MOVING TOWARDS A NEW GOLD STANDARD?}

${ }^{1}$ Ahmir Ahmad*, ${ }^{1}$ Ana Wilson, ${ }^{2}$ Morgan Moorghen, ${ }^{1}$ Angad Dhillon, 'Siwan ThomasGibson, 'Noriko Suzuki, ${ }^{1}$ Adam Humphries, ${ }^{1}$ Adam Haycock, ${ }^{1}$ Kevin Monahan, ${ }^{1}$ Margaret Vance, 'Brian Saunders. 'Wolfson Unit for Endoscopy, Imperial College London, London, UK; ${ }^{2}$ Pathology Department, St Mark's Hospital, London, UK

\subsection{6/gutjnl-2021-BSG.50}

Introduction Histopathology is regarded as the gold standard for diagnosis of small colonic polyps. However, there is growing interest in optical diagnosis and implementation of a 'resect and discard' strategy. Our aim is to evaluate accuracy of histopathology reporting where a high confidence diminutive polyp optical diagnosis was made and to assess the impact of performing additional tissue section re-cuts, where there is a discrepancy.

Methods Eight bowel cancer screening colonoscopists optically diagnosed 639 diminutive polyps during the period Feb-Nov 2020 in the early phase of a prospective feasibility study of optical diagnosis (DISCARD3). Each polyp diagnosis was evaluated by the colonoscopist as high or low confidence. All retrieved polyps were sent for histopathology.

Discrepancy between high confidence optical diagnoses and histopathology were re-reported by a second pathologist blinded to the original optical and histological call. If discrepancy remained after re-review, the polyp was re-cut into deeper levels and a third blinded histopathology review performed.

Results Of 639 diminutive polyps, 468 (73.2\%) were high confidence optical calls and 171 (26.8\%) were low confidence (see figure 1).

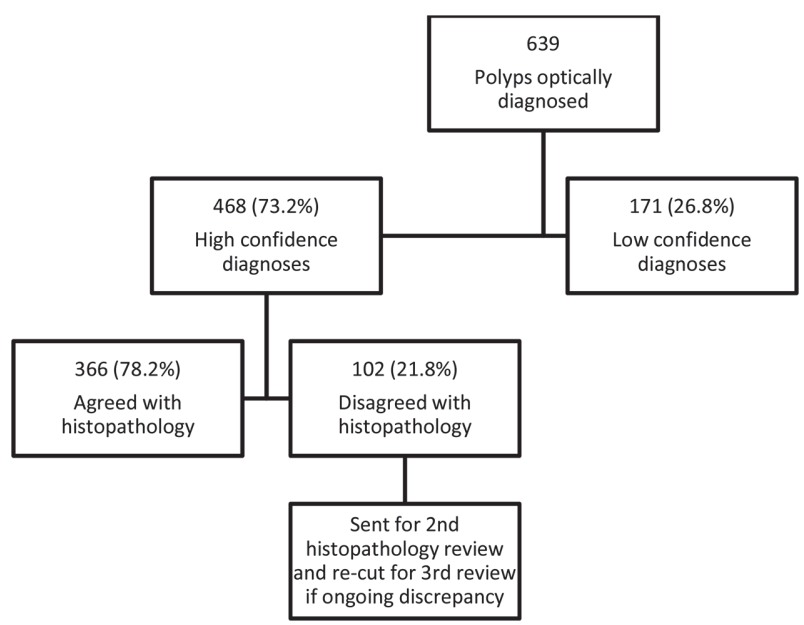

Abstract HTU-3 Figure 1 Overview of study 